\title{
Factores de riesgo y precursores del Deterioro Cognitivo Leve (DCL): Una mirada sinóptica
}

\author{
Anticipatory signs and risk factors for Mild Cognitive Impairment (MCI): \\ A synoptic view.
}

\author{
Margarita Cancino \\ Lucio Rehbein \\ Universidad de La Frontera, Chile
}

Rec. (24 de diciembre de 2015) Acept. (13 de noviembre de 2016)

\begin{abstract}
Resumen
Este artículo presenta una breve reseña sobre el Deterioro Cognitivo Leve en adultos mayores, considerando los principales precursores que anuncian, y los factores de riesgo que aceleran los procesos de envejecimiento cognitivo, los cuales aumentan la probabilidad de recibir un diagnóstico de DCL. A su vez, la presencia de DCL aumenta el riesgo y eventual tránsito hacia la demencia, especialmente la demencia de tipo Alzheimer. Por otra parte, en el artículo se describen las diferentes modalidades de presentación del deterioro cognitivo, revisando también el DCL asociado a la sintomatología depresiva, a la enfermedad de Parkinson y otras enfermedades degenerativas del SNC. La presente revisión, permite dar cuenta de hallazgos recientes y relevantes con relación a la disfunción cognitiva en adultos mayores, quienes representan una proporción cada vez mayor de la población nacional y mundial.

Palabras clave: envejecimiento, deterioro cognitivo leve, indicadores tempranos, demencia
\end{abstract}

\begin{abstract}
This article presents a brief overview of mild cognitive impairment in older adults, considering its main anticipating precursors, and the risk factors that may accelerate the processes of cognitive aging, which increases the likelihood of being diagnosed with MCI. In turn, the presence of MCL increases the risk and eventual transition to dementia, particularly Alzheimer's dementia. Moreover, the article describes the different forms cognitive impairment may present itself, and also reviews MCI associated with depressive symptoms, Parkinson's disease and other degenerative diseases of the CNS. This revision accounts for recent and relevant findings regarding cognitive dysfunction in the elderly, who represent a growing share of national and global populations.

Key words: Aging; mild cognitive impairment; psychological precursors, indices of MC
\end{abstract}

\footnotetext{
Correspondencia: Margarita Cancino Andrade, Doctorado en Psicología, Universidad de La Frontera, Avenida Francisco Salazar 01145. Temuco, Chile. Dirección electrónica: margarita.cancino@ufrontera.cl, Teléfono: 45-2325620.

Nota de autor. Margarita Cancino, DrC. Doctorado en Psicología, Universidad de La Frontera, Chile.

Lucio Rehbein, Ph.D. Departamento de Psicología, Universidad de La Frontera, Chile
} 


\section{Introducción}

Dos de los cambios importantes ocurridos durante las últimas décadas son el aumento considerable en la expectativa de vida de la población y la reducción de la tasa de natalidad. Esto ha traído consigo un considerable envejecimiento de la población. Para las Naciones Unidas, un país se encuentra envejecido cuando el $7 \%$ o más de su población tiene más de 60 años de edad (Banco Mundial, 2013, Mathers et al. 2015). Sin embargo, Chile ha superado con creces ese nivel, con tasas actuales superiores al $13.5 \%$ de adultos mayores de 60 años y con una proyección de llegar al $20 \%$ en el año 2025 (SENAMA).

El creciente estudio de los cambios y características de este segmento de la población, ha permitido concluir que las enfermedades asociadas al envejecimiento son diversas y se traducen en un deterioro de la calidad de vida de quienes las padecen y de sus familias. Además, la atención de estas enfermedades generan un alto costo económico para los servicios de salud (Marín, 2007). Un estudio reciente de Prince y colaboradores (2015) señala que a nivel mundial, el $23 \%$ del gasto en salud está destinado a la atención de enfermedades en el segmento de adultos mayores de 60 años y que el $7 \%$ de ese gasto corresponde a enfermedades neurológicas y mentales (Prince et al., 2015).

Dentro de la diversidad de patologías asociadas al envejecimiento, se encuentra el deterioro cognitivo leve (DCL), cuadro que se manifiesta como un conjunto de alteraciones en las funciones cognitivas básicas, esto es; orientación espacial, lenguaje, reconocimiento visual, y un predominio de la reducción de la función mnémica. Además, se suelen acompañar cambios conductuales (Barrera, Donolo, y Rinaudo, 2010).

Para algunos autores, el DCL es considerado un posible precursor de las demencias, colocándolo como una etapa intermedia entre el envejecimiento normal y la demencia, más específicamente, de la demencia de tipo Alzheimer (Donoso y Vásquez, 2002). Sin embargo, una revisión de la literatura publicada recientemente por Forlenza y colaboradores (2013), da cuenta que un sujeto con síntomas compatibles con el deterioro cognitivo, eventualmente podría avanzar hacia el desarrollo de algún tipo de demencia, permanecer estable, e incluso, con la intervención adecuada, podría retomar su funcionamiento cognitivo normal (Forlenza, Diniz, Stella, Teixeira y Gattaz, 2013).

\section{Origen y trayectorias del DCL}

Una de las primeras definiciones conceptuales de este cuadro surgió de la necesidad de interpretar un rango intermedio de los resultados obtenidos con la administración de la Escala de Deterioro Global de Flicker, Ferris, y Reisberg (1991). Por su parte Petersen, uno de los autores vastamente citados en este ámbito, se refiere al deterioro cognitivo leve como un síndrome que puede presentar déficits cognitivos por sobre lo que se espera a una cierta edad en una determinada cultura (Petersen y Morris, 2005).

La conceptualización del deterioro cognitivo como un estado transicional entre los procesos de cambio propios del envejecimiento y un estadio temprano de la demencia, se ha transformado en un ámbito de interés para los investigadores básicos y clínicos (Pose y Mares, 2010; Forlenza et al., 2013), llevándolos a realizar estudios clínicos y epidemiológicos de tipo longitudinal, para monitorear el funcionamiento cognitivo de las personas de la tercera edad.

Estos estudios han encontrado que los sujetos que presentan un mayor rendimiento cognitivo global, en las mediciones iniciales, tienen menos propensión a desarrollar demencia de tipo Alzheimer. En contraposición, los sujetos de mayor edad que presentan un bajo rendimiento global en las mediciones iniciales, aumentan el riesgo de desarrollar demencia de tipo Alzheimer (Rossini, Rossi, Babiloni, y Polich, 2007; Forlenza et al., 2013).

El deterioro cognitivo comparte con la demencia factores de riesgo como la edad, el sexo, el nivel educativo, la carga genética y la presencia de trastornos depresivos (Campbell, Unverzagt, LaMantia, Khan, y Boustani, 2013).

Adicionalmente, dado que, se sabe que por sobre los 60 años, aumenta el riesgo de padecer DCL (López y Calero, 2009) y que las mujeres tienen un riesgo mayor de padecer Demencia de tipo Alzheimer, toda investigación en este ámbito debe considerar (o controlar) estos factores de riesgo (Mackin, Insel, Aisen, Geda, y Weiner, 2012; Sánchez y Torrellas, 2011).

Por otra parte cabe señalar que las quejas de memoria no sólo responden al DCL, sino también suelen ser explicadas por la ansiedad y la depresión (Donoso (2007). En esta misma línea, en un estudio transversal realizado por Baquero et al. (2004), se encontró que los síntomas psicológicos y conductuales que tienen mayor asociación con el DCL son: depresión, irritabilidad, ansiedad, apatía y agitación.

Diferentes investigaciones reportan la importancia de construir perfiles de ansiedad y depresión en sujetos de avanzada edad, para determinar su relación con los diferentes tipos de DCL (Andreescu, Teverovsky, Hughes, Chang y Ganguli, 2014; Sacuiu et al., 2015).

La ansiedad y la depresión tienen una relación negativa con el bienestar, alterando las relaciones interpersonales y el apoyo social percibido del sujeto que las padece. Una 
investigación acerca del bienestar psicológico en adultos mayores, reportó que el optimismo es un predictor del bienestar psicológico. Adicionalmente, el apoyo social percibido actúa como mediador de la relación entre el optimismo y el bienestar subjetivo (Ferguson y Goodwin, 2010). Por otra parte el optimismo ha sido identificado como moderador de la relación entre el estrés psicológico y la sintomatología depresiva (Marquez-González, Baltar, Puente, y RomeroMoreno, 2009; Vera-Villarroel, Pavez, y Silva, 2012).

\section{Tipos de Deterioro Cognitivo}

En la actualidad, no sólo se distinguen el DCL amnésico y el no amnésico; esto es, el predominio, o no, del deterioro en la función mnémica, sino que también se ha logrado establecer una diferenciación según el número de dominios afectados, es decir, habría sujetos con DCL unidominio, cualquiera sea éste; y otros con DCL multidominio, donde existiría más de un dominio cognitivo deteriorado. Estas clasificaciones buscan facilitar una mayor especificidad en la detección y diagnóstico del DCL.

Con el objetivo de alcanzar una mayor especificidad y validez ecológica, en la Universidad Estatal de Washington, un equipo de investigadores utilizó un departamento del campus para observar, con tecnologías no intrusivas, la ejecución de tareas de la vida cotidiana, en sujetos cognitivamente sanos y sujetos con DCL unidominio y multidomino. Los resultados indicaron que, en ambientes naturales, los participantes con DCL multidominio tienen mayores dificultades para completar con precisión las tareas solicitadas, que los sujetos con DCL de un solo dominio, cuyo rendimiento resultó bastante similar al de los adultos mayores sin compromiso cognitivo (Seelye, Schmitter-Edgecombe, Cook, y Crandall, 2013).

Diversas investigaciones orientadas a identificar los síntomas o principales cambios que se generan a nivel cognitivo en sujetos con DCL, han encontrado que la alteración a nivel mnémico es el principal indicador de disfunción. Petersen y Morris (2005) establecieron dos tipos de DCL: uno de tipo amnésico y otro de tipo no amnésico, categorización que es independiente de la cantidad de dominios cognitivos afectados por la enfermedad; esto es, uni o multidominio.

La investigación de Farías y su equipo (Farías et al, 2006), da cuenta que las personas con DCL presentan dificultades para realizar exitosamente actividades instrumentales de la vida cotidiana, es decir, tareas que tienen cierta complejidad cognitiva, como por ejemplo; cocinar, regular sus finanzas o manejar su medicación (Seelye et.al., 2013).

En concreto, la evidencia de disfunción cognitiva estará dada, en primera instancia, por el tipo de DCL, sin embargo, existe un alto grado de acuerdo en que las principales funciones cognitivas que se alteran son: la memoria episódica, la cual se ha relacionado con la fase prodrómica de la demencia de tipo Alzheimer y con el DCL de tipo amnésico, según la clasificación de Petersen. Este tipo de memoria, forma parte de la clasificación clásica que realizaron Tulving y Schacter (1990), bajo los criterios de: áreas cerebrales involucradas, tipo de información procesada y los principios de sus operaciones, en su definición. Estos autores postulan que la memoria episódica correspondería a la recolección consciente que un sujeto realiza de su pasado personal (Tulving y Schacter, 1990).

\section{Biomarcadores del Deterioro Cognitivo}

Los biomarcadores son variables bioquímicas, fisiológicas y/o anatómicas que son medibles, in situ, con el objetivo de identificar características específicas de una enfermedad o diferenciarla de otra (Jack et al., 2010).

En la actualidad, diversas investigaciones incluyen marcadores biológicos y neurales para estudiar el funcionamiento cognitivo (Valls-Pedret, Molinuevo y Rami, 2010). El avance en las técnicas de exploración cerebral, ha permitido el desarrollo de líneas de investigación muy específicas en torno al DCL y la Demencia de tipo Alzheimer (Binney, Embleton, Jefferies, Parker y Ralph, 2010).

Una técnica utilizada para explorar el funcionamiento cerebral es el electroencefalograma (EEG). Al respecto es posible afirmar que el patrón de un EEG, en sujetos que están en etapas tempranas de la enfermedad de Alzheimer, se caracteriza por un incremento en la actividad de ondas theta $\mathrm{y}$ una disminución en la actividad beta, y posteriormente, en la actividad de ondas alfa, en tanto que el aumento en las ondas delta sería esperable en los estados más avanzados de la enfermedad (Babiloni et al., 2004).

Estos indicadores serían similares en sujetos con DCL, sin embargo, existen divergencias empíricas por cuanto, en algunos estudios, estas variaciones espectrales permiten distinguir bien a los sujetos con DCL de sujetos con demencia de tipo Alzheimer, pero se torna más difícil al momento de querer diferenciar a los sujetos sanos de aquellos con DCL (Fernández et al., 2012).

Forlenza y colaboradores (2013), se refieren a las técnicas de neuroimagen como la medida menos invasiva para indagar en los cambios que diferentes patologías generan en el cerebro. En relación con el DCL, estos autores afirman que la resonancia magnética, así como las tomografías por emisión de positrones, son medios importantes para detectar, a través de estudios longitudinales, los cambios 
estructurales y la pérdida de volumen en áreas específicas del cerebro como el hipocampo y una disminución del grosor de la corteza cerebral (Forlenza, Diniz, Stella, Teixeira y Gattaz, 2013). Es posible afirmar que la caracterización de estos cambios constituye un importante biomarcador para detectar a sujetos en riesgo de deterioro cognitivo y realizar diagnósticos más pertinentes.

Entre los biomarcadores del DCL se encuentran la proteína TAU y el péptido beta amiloide 42. La saturación o hiperfosforilación de la proteína (P-TAU), favorece la formación de ovillos neurofibrilares, los que afectan principalmente a los lóbulos temporales mediales y a las estructuras corticales asociativas. El péptido beta amiloide, por su parte, es el componente esencial de las placas neuríticas, el principal marcador de neurodegeneración cerebral. A nivel fisiológico, la producción defectuosa o excesiva de este péptido, origina procesos de reacciones inflamatorias localizadas y cambios a nivel neuronal como consecuencia de la fosforilación de la proteína TAU, lo que eventualmente podría causar disfunción y muerte neuronal (Hansson et al., 2010; Mrak, 2009)

En la utilización de biomarcadores, se ha desarrollado el concepto de reserva cerebral, que se traduce en la medición del tamaño del cerebro y el recuento neuronal. Diversas investigaciones han demostrado que los sujetos con patologías cerebrales, presentan indicadores de menor reserva cerebral que los sujetos sanos, controlando por edad (Borroni et al., 2008; Díaz-Orueta, Buiza-Bueno, y Yanguas-Lezaun, 2010; Fratiglioni y Wang, 2007; Satz, 1993).

\section{Factores sociodemográficos y de estilo de vida asociados al Deterioro Cognitivo Leve}

Además de los factores psicológicos y neurobiológicos recién revisados, los antecedentes teóricos y empíricos, dan cuenta también, de variables sociodemográficas y experiencias de vida que influenciarían el funcionamiento cognitivo de los adultos mayores (Brewster et al., 2014).

En este contexto Glymour y Manly (2008), plantean que circunstancias vitales de la temprana y mediana edad, podrían modificar el funcionamiento cognitivo en la adultez mayor, por la influencia de los patrones de estilo de vida, los que a su vez se correlacionan de manera directa con el nivel socioeconómico, variable que se asocia al nivel educativo y la etnia, resultando complejo delimitar el real influjo de cada variable (Brewster et al., 2014).

Diferentes investigaciones han detectado asociaciones positivas entre el envejecimiento cognitivo y el nivel educativo, concluyendo que la calidad y la duración de la escolarización influyen de manera directa en el funcionamiento cognitivo global y en la reserva cognitiva (Allegri et al., 2010); Brewster et al., 2014). Un estudio reciente realizado por Meléndez - Moral y su equipo (2012) concluyó que los sujetos con más edad y menos años de escolarización, tienen mayor riesgo de cumplir con los criterios de inclusión para el diagnóstico de DCL; su muestra estuvo constituida por sujetos de entre 60 y 89 años de edad. Adicionalmente, los años de escolaridad tienen un correlato con el tipo de actividad laboral que desarrolla una persona, variable que también se incluye en los modelos de predicción del DCL (Lojo-Seoane, Facal, y Juncos-Rabadán, 2012).

Otras variables que han sido exploradas en su relación con el funcionamiento cognitivo, son las actividades recreativas y de ocio (Brewster et al., 2014). Un estudio inicial acerca del riesgo de Demencia desarrollado en China, demostró que las actividades comunitarias y recreativas, como la jardinería, eran un factor protector en la incidencia de demencia (Zhang y Zhang, 1999). Estudios más recientes dan cuenta de que un estilo de vida activo, esto es, actividad cognitiva (leer, pintar, escribir, hacer crucigramas), actividad física (practicar algún deporte o caminar) y actividades sociales (participación en clubes, iglesias), practicadas con regularidad, pueden retrasar la aparición del deterioro cognitivo (Arenaza-Urquijo et al., 2011; Verghese, 2006; Wilson, 2010).

Buscando una mayor precisión en la comprensión del funcionamiento cognitivo, Brewster et al., (2014) realizó un estudio longitudinal en el que se examinó la influencia de las experiencias de vida y los factores demográficos en el funcionamiento cognitivo de adultos mayores. Ellos encontraron que la edad, la alfabetización, el nivel socioeconómico en la niñez, y la actividad física, tienen una influencia significativa en el funcionamiento cognitivo (Brewster et al., 2014).

En suma, diferentes investigaciones han encontrado que la edad y la disminución de la actividad intelectual y social a partir de los 40 años, se asocian de manera independiente del nivel socioeconómico y la alfabetización, con un aumento del deterioro cognitivo en la tercera edad. En esta misma línea, los hallazgos dan cuenta que aquellos sujetos que cursaron estudios superiores y se mantienen realizando actividad física en la mediana edad, tienen menor riesgo de declive cognitivo, o éste se presenta con una progresión más lenta (Brewster et al., 2014; Melrose et al., 2015; Reed et al., 2011; Schiepers et al., 2012; Wilson et al., 2012).

\section{Deterioro cognitivo y Demencia de tipo Alzheimer}

Se ha comprobado empíricamente que un 15 a $20 \%$ del deterioro cognitivo de tipo amnésico, tiene un alto correlato 
con el diagnóstico posterior de demencia del tipo Alzheimer, en un lapso de tiempo que puede variar de los dos a los seis años (Guerra, 2012; Samper, Llibre, Sosa y Solórzano, 2011; Conde-Sala et al., 2013).

El correlato del deterioro cognitivo leve con el posterior diagnóstico de demencia de tipo Alzheimer, ha sido indagado en diversas investigaciones. Recientemente, SchmitterEdgecombe y Parsey (2014), concluyeron que en el DCL y la Demencia de tipo Alzheimer inicialmente, los sujetos presentan dificultades para: vestirse, manejar adecuadamente el dinero y/o realizar actividades de la vida cotidiana como cocinar o conducir un automóvil. Sin embargo, medir estas variables en la clínica y/o en el laboratorio requiere, por lo general, el reporte de informantes que pueden ser familiares o cuidadores; pero, en contextos de investigación se ha encontrado que los reportes de familiares y cuidadores, no siempre se correlacionan con las evaluaciones de rendimiento cognitivo que se administran al propio sujeto (Jefferson et al., 2008; Schmitter-Edgecombe, Parsey, y Cook, 2011).

\section{Deterioro Cognitivo y enfermedad de Parkinson}

La enfermedad de Parkinson, que corresponde a una alteración neurodegenerativa en la que existe un predominio de síntomas motores, ha sido sindicada como el trastorno más común y la segunda enfermedad neurodegenerativa de mayor prevalencia después de la Demencia de tipo Alzheimer. Cifras globales permiten estimar que un 1 a 2 $\%$ de la población de personas mayores de 65 años tiene la enfermedad de Parkinson (Alves, Forsaa, Pedersen, Dreetz-Gjerstad y Larsen, 2008; Wirdefeldt, Adami, Cole, Trichopoulos, y Mandel, 2011).

Los síntomas característicos de la enfermedad son: disfunción motora, especialmente temblor en reposo, bradicinesia, rigidez y alteración del reflejo postural (Wirdefeldt et al., 2011). Adicionalmente, puede haber presencia de alteración cognitiva, específicamente en la función ejecutiva, habilidades visoespaciales y la memoria (Janvin, Larsen, Aarsland, y Hugdahl, 2006).

Diversas investigaciones han encontrado evidencia de la relación entre la demencia y la enfermedad de Parkinson, sin embargo, la asociación entre deterioro cognitivo y la enfermedad de Parkinson ha sido menos investigada, presumiblemente por el solapamiento y la dificultad diagnóstica que se genera con otros trastornos como, por ejemplo, la depresión, ya que ésta empeoraría el rendimiento de los sujetos en tareas que midan rendimiento en atención y función ejecutiva, procesos cognitivos que se evalúan para hacer el diagnóstico de Deterioro Cognitivo leve (ToribioDíaz y Carod-Artal, 2015).

En un estudio realizado en el Hospital Universitario de Stavanger, donde participaron 72 sujetos, se encontró que un $62 \%$ de los pacientes diagnosticados con Deterioro Cognitivo Leve y Enfermedad de Parkinson progresaron hacia la demencia en un período de 4 años, en cambio de los sujetos con enfermedad de Parkinson, pero intactos cognitivamente, sólo un 20\% desarrollaron Demencia, (Janvin, Larsen, Aarsland, y Hugdahl, 2006) estos hallazgos evidencian lo progresivo de la disfunción cognitiva y refuerzan la asociación entre el Deterioro Cognitivo Leve y la Demencia de tipo Alzheimer.

\section{Conclusiones}

A partir de los antecedentes antes expuestos, es posible afirmar que los estudios sobre envejecimiento, deben procurar considerar los factores cognitivos y su influencia en el funcionamiento del adulto mayor, ya que los signos iniciales de disfunción cognitiva, no aparecen exclusivamente en la adquisición y recuperación de información, sino también en la capacidad de organización y, en algún sentido también, afectan el funcionamiento en la vida cotidiana.

El Deterioro Cognitivo, se asocia con otros trastornos como la depresión o la presencia de sintomatología depresiva, por lo tanto se estima que la progresión del deterioro cognitivo puede ser secundaria a la presencia de depresión. En consecuencia, es necesario que investigadores y clínicos incluyan en su evaluación el screening de depresión, utilizando instrumentos diseñados para estos fines, exclusivos para adultos mayores, considerando la presencia de otras enfermedades como por ejemplo la enfermedad de Parkinson que también se asocia con DCL y con depresión, y que en presencia de enfermedad de Parkinson y DCL, aumenta considerablemente el riesgo de progresión hacia la demencia.

Respecto a considerar el DCL como un factor de tránsito a la demencia, es importante destacar que el desarrollo de estudios longitudinales, contribuirá a la comprensión del concepto de trayectoria de envejecimiento y disfunción cognitiva, además es importante destacar que no se debe unificar la disfunción cognitiva del adulto mayor, sólo en los déficit de la memoria, pues existe suficiente evidencia para afirmar que otros dominios cognitivos como la función ejecutiva, la atención y la percepción espacial, se ven implicados en la reducción del rendimiento cognitivo.

Con los antecedentes antes expuestos, es urgente y necesario incrementar los estudios neuropsicológicos basales 
y de seguimiento a los adultos mayores, a fin de identificar y medir los eventuales riesgos de desarrollar DCL. La evaluación neuropsicológica es la mejor estrategia para detectar disfunción cognitiva y ésta debe considerar la influencia que variables como la edad, nivel educativo y el estado afectivo tienen en el rendimiento de un sujeto. La evaluación y seguimiento de los adultos mayores, permitiría determinar el tipo de deterioro cognitivo, evaluar su potencial eventual tránsito hacia la demencia, y eventualmente, a partir de la evaluación, generar la intervención adecuada.

Finalmente, teniendo en consideración los avances de la investigación especializada revisados en las secciones anteriores, es necesario destacar que el estado de avance logrado destaca la importancia que reviste la preservación del funcionamiento neurocognitivo en el proceso de envejecer, y que los esfuerzos de investigadores y clínicos deben centrarse en la identificación de los factores de riesgo para generar medidas preventivas inclusivas y eficaces, que contribuyan a mejorar la calidad de vida de los adultos mayores.

\section{Referencias}

Allegri, R. F., Taragano, F. E., Krupitzki, H., Serrano, C. M., Dillon, C., Sarasola, D., y

Sánchez, V. (2010). Role of cognitive reserve in progression from mild cognitive impairment to dementia. Dementia \& Neuropsychologia, 4(1), 28-34.

Alves, G., Forsaa, E. B., Pedersen, K. F., Gjerstad, M. D., y Larsen, J. P. (2008). Epidemiology of Parkinson's disease. Journal of neurology, $255,18-32$

Andreescu, C., Teverovsky, E., Fu, B., Hughes, T. F., Chang, C. C. H., y Ganguli, M. (2014). Old worries and new anxieties: behavioral symptoms and mild cognitive impairment in a population study. The American Journal of Geriatric Psychiatry, 22, 274-284.

Arenaza-Urquijo, E.M., Bosch, B., Sala-Llonch, R., Solé-Padullés ,C., Junqué, C., ... Fernández-...Espejo, D., (2011). Specific anatomic associations between white matter integrity and cognitive reserve in normal and cognitively impaired elders. The American Journal of Geriatric Psychiatry, 19, 33-42.

Babiloni, C., Binetti, G., Casseta, E., Cerboneschi, D., Dal Forno, G., ... Del Percio, C. (2004). Mapping distributed sources of cortical rythms in mild Alzheimer's disease. Amulticentric EEG study. Neuroimage. 22, 57- 67.

Banco Mundial (2013). Sección datos de libre acceso. Recuperado desde http://datos.bancomundial.org/

Baquero, M., Blasco, R., Campos-García, A., Garcés, M., Fages, E. M., y Andreu-Català, M. (2004). Estudio descriptivo de los trastornos conductuales en el deterioro cognitivo leve. Revista de Neurología, 38, 323-326.

Barrera, M., Donolo, D., y Rinaudo, M. C. (2010). Riesgo de demencia y niveles de educación: Cuando aprender es más saludable de lo que pensamos. Anales de psicología, 26, 34-40.

Binney, R. J., Embleton, K. V., Jefferies, E., Parker, G. J., y Ralph, M. A. L. (2010). The ventral and inferolateral aspects of the anterior temporal lobe are crucial in semantic memory: evidence from a novel direct comparison of distortion-corrected fMRI, rTMS, and semantic dementia. Cerebral Cortex, 20, 2728-2738.

Borroni, B., Premi, E., Agosti, C., Alberici, A., Garibotto, V., Bellelli, G., y Padovani, A. (2008). Revisiting brain reserve hypothesis in fronto- temporal dementia: evidence from a brain perfusion study. Dementia and geriatric cognitive disorders, 28, 130-135.

Brewster, P. W., Melrose, R. J., Marquine, M. J., Johnson, J. K., Napoles, A., MacKay-Brandt, A., Farias, S., Reed, B., y Mungas, D. (2014). Life Experience and Demographic Influences on Cognitive Function in Older Adults. Neuropsychology, 28, 846.

Campbell, N. L., Unverzagt, F., LaMantia, M. A., Khan, B. A., y Boustani, M. A. (2013). Risk Factors for the Progression of Mild Cognitive Impairment to Dementia. Clinics in geriatric medicine, 29, 873-893.

Conde-Sala, J. L., Garre-Olmo, J., Vilalta-Franch, J., Llinàs-Reglà, J., Turró-Garriga, O., Lozano-Gallego, M., y López-Pousa, S. (2013). Declive cognitivo en la enfermedad de Alzheimer. Seguimiento de más de tres años de una muestra de pacientes. Revista de Neurología, 56, 593-600.

Díaz-Orueta, U., Buiza-Bueno, C., y Yanguas-Lezaun, J. (2010). Reserva cognitiva: evidencias, limitaciones y líneas de investigación futura. Revista Española de Geriatría y Gerontología, 45, 150-155.

Donoso, A., y Vásquez, C. (2002). Deterioro Cognitivo y Enfermedad de Alzheimer: Presentación de dos Casos. Revista de Psicología, 11, 9 -15.

Donoso, A., (2007). Deterioro y demencia: Orientación para médicos no especialistas. Cuadernos de neuropsicología, 1, 115-126.

Farias, S. T., Mungas, D., Reed, B. R., Harvey, D., Cahn-Weiner, D., y DeCarli, C. (2006). MCI is associated with deficits in everyday functioning. Alzheimer disease and associated disorders, 20(4), 217.

Ferguson, S. J., y Goodwin, A. D. (2010). Optimism and well-being in older adults: The mediating role of social support and perceived control. The International Journal of Aging and Human Development, 71, 43-68.

Fernández, A., Turrero, A., Zuluaga, P., Gil-Gregorio, P., del Pozo, F., Maestu, F., y Moratti, S. (2012). MEG delta mapping along the healthy aging-Alzheimer's disease continuum: diagnostic implications. Journal of Alzheimer's disease, 35, 495-507.

Flicker, C., Ferris, S. H., y Reisberg, B. (1991). Mild cognitive impairment in the elderly predictors of dementia. Neurology, 41, 1006-1006.

Forlenza, O. V., Diniz, B. S., Stella, F., Teixeira, A. L., y Gattaz, W. F. (2013). Mild cognitive impairment (part 1): clinical characteristics and predictors of dementia. Revista Brasileira de Psiquiatria, 35, 178-185.

Fratiglioni, L., y Wang, H. X. (2007). Brain reserve hypothesis in dementia. Journal of Alzheimer's disease, 12, 11-22.

Glymour, M. M., y Manly, J. J. (2008). Lifecourse social conditions and racial and ethnic patterns of cognitive aging. Neuropsychology review, $18,223-254$.

Guerra, M. (2012). Enfermedad de Alzheimer:¿Es posible la prevención? Palabras Mayores, 1(2), 1 - 9 .

Hansson, O., Zetterberg, H., Vanmechelen, E., Vanderstichele, H., Andreasson, U., Londos, E., ... y Blennow, K. (2010). Evaluation of plasma $A \beta 40$ and $A \beta 42$ as predictors of conversion to Alzheimerss disease in patients with mild cognitive impairment. Neurobiology of aging, 31(3), 357-367.

Jack, Jr. C. R., Knopman, D. S., Jagust, W. J., Shaw, L. M., Aisen, P. S., Weiner, M. W., y Trojanowski, J. Q. (2010). Hypothetical model of dynamic biomarkers of the Alzheimer's pathological cascade. The Lancet Neurology, 9, 119-128.

Janvin, C. C., Larsen, J. P., Aarsland, D., y Hugdahl, K. (2006). Subtypes of mild cognitive impairment in Parkinson's disease: progression to dementia. Movement Disorders, 21, 1343-1349.

Jefferson, A. L., Byerly, L. K., Vanderhill, S., Lambe, S., Wong, S., Ozonoff, A., y Karlawish, J. H. (2008). Characterization of activities of daily living in individuals with mild cognitive impairment. The American Journal of Geriatric Psychiatry, 16, 375-383.

Lojo-Seoane, C., Facal, D., y Juncos-Rabadán, O. (2012). ¿Previene la actividad intelectual el deterioro cognitivo? Relaciones entre reserva cognitiva y deterioro cognitivo ligero. Revista Española de Geriatría y Gerontología, 47, 270-278.

López, Á. G., y Calero, M. D. (2009). Predictores del deterioro cognitivo en ancianos.Revista Española de Geriatría y Gerontología, 44, 220-224.

Mackin, R. S., Insel, P., Aisen, P. S., Geda, E., y Weiner, M. W. (2012). Longitudinal stability of subsyndromal symptoms of depression in individuals with mild cognitive impairment: relationship to conversion 
to dementia after 3 years. International Journal of Geriatric Psychiatry, 27, 355-363.

Marín, P. P. (2007). Reflexiones para considerar en una política pública de salud para las personas myores: Thoughts for action. Revista médica de Chile, 135, 392-398.

Márquez-González, M., Baltar, A. L., Puente, C. P., y Romero-Moreno, R. (2009). El optimismo como factor moderador de la relación entre el estrés y la depresión de los cuidadores informales de personas mayores dependientes. Revista Española de Geriatría y Gerontología, 44(5), 251-255.

Mathers, C. D., Stevens, G. A., Boerma, T., White, R. A., y Tobias, M. I. (2015). Causes of international increases in older age life expectancy. The Lancet, 385,540-548.doi:http://dx.doi.org/10.1016/S01406736(14)60569-9

Meléndez-Moral, J. C., Sanz-Álvarez, T., y Navarro-Pardo, E. (2012). Deterioro cognitivo leve: método y procedimiento de clasificación. Anales de psicología, 28(2), 604-610.

Melrose, R. J., Brewster, P., Marquine, M. J., MacKay-Brandt, A., Reed, B., Farias, S. T., y Mungas, D. (2015). Early life development in a multiethnic sample and the relation to late life cognition. The Journals of Gerontology Series B: Psychological Sciences and Social Sciences, $70,519-531$

Mrak, R. E. (2009). Neuropathology and the neuroinflammation idea. Journal of Alzheimer's Disease, 18, 473-481.

Petersen, R. C., y Morris, J. C. (2005). Mild cognitive impairment as a clinical entity and treatment target. Archives of Neurology, 62, 1160-1163.

Pose, M., y Mares, F. (2010). Instituto de Neurología Cognitiva (INECO). Instituto de Neurociencias de la Fundación Favaloro, Buenos Aires Argentina.

Prince, M. J., Wu, F., Guao, Y., Gutiérrez, L., O’Donell, M., Sullivan, R., y Yusuf, S.(2015). The burden of disease in older people and implications for health policy and practice. The Lancet, 385, 548-556. doi: http:// dx.doi.org/10.1016/S0140-6736(14)61347-7

Reed, B. R., Dowling, M., Tomaszewski Farias, S., Sonnen, J., Strauss, M., Schneider, J. A., y Mungas, D. (2011). Cognitive activities during adulthood are more important than education in building reserve. Journal of the International Neuropsychological Society, 17, 615-624.

Rossini, P. M., Rossi, S., Babiloni, C., y Polich, J. (2007). Clinical neurophysiology of aging brain: from normal aging to neurodegeneration. Progress in neurobiology, 83, 375-400.

Sacuiu, S., Insel, P. S., Mueller, S., Tosun, D., Mattsson, N., Jack, C. R., DeCarli, C., Petersen, R., Aisen, P.S., Weiner, M.W., y Mackin, R. S. (2015) Alzheimer's Disease Neuroimaging Initiative (ADNI). Chronic Depressive Symptomatology in Mild Cognitive Impairment is Associated with Frontal Atrophy Rate which Hastens Conversion to Alzheimer Dementia. The American Journal of Geriatric Psychiatry. doi:10.1016/j.jagp.2015.03.006

Samper Noa, J. A., Llibre Rodríguez, J. J., Sosa Pérez, S., y Solórzano Romero, J. (2011). Del envejecimiento a la enfermedad de alzheimer. Revista Habanera de Cienciasispép:Médicas, 10(3), 319-327

Sánchez, J., y Torrellas, C. (2011). Revisión del constructo deterioro cognitivo leve: aspectos generales. Revista de Neurología, 52, 300-305.

Satz, P. (1993). Brain reserve capacity on symptom onset after brain injury: a formulation and review of evidence for threshold theory. Neuropsychology, 7, 273.

Schiepers, O. J. G., Harris, S. E., Gow, A. J., Pattie, A., Brett, C. E., Starr, J. M., y Deary, I. J. (2012). APOE E4 status predicts age-related cognitive decline in the ninth decade: longitudinal follow-up of the Lothian Birth Cohort 1921. Molecular psychiatry, 17, 315-324.

Schmitter-Edgecombe, M., y Parsey, C. M. (2014). Assessment of functional change and cognitive correlates in the progression from healthy cognitive aging to dementia. Neuropsychology, 28, 881 .

Schmitter-Edgecombe, M., Parsey, C., y Cook, D. J. (2011). Cognitive correlates of functional performance in older adults: Comparison of selfreport, direct observation, and performance-based measures. Journal of the International Neuropsychological Society, 17, 853-864.

Seelye, A. M., Schmitter-Edgecombe, M., Cook, D. J., y Crandall, A. (2013). Naturalistic assessment of everyday activities and prompting technologies in mild cognitive impairment. Journal of the International Neuropsychological Society, 19, 442-452.

Servicio Nacional del Adulto Mayor. (2009). Estudio Nacional de la Dependencia en las Personas Mayores. Disponible en http://www.senama. cl/filesapp/Estudio_dependencia.pdf

Toribio-Díaz, M. E., y Carod-Artal, F. J. (2015). Subtipos de deterioro cognitivo leve en la enfermedad de Parkinson y factores predictores de conversión a demencia. Revista de Neurología, 14, 24.

Tulving, E., y Schacter, D. L. (1990). Priming and human memory systems. Science, 247, 301-306.

Valls-Pedret, C., Molinuevo, J. L., y Rami, L. (2010). Diagnóstico precoz de la enfermedad de Alzheimer: fase prodrómica y preclínica. Neurología, 51, 471-480.

Vera-Villarroel, P., Pávez, P., y Silva, J. (2012). El rol predisponente del optimismo: hacia un modelo etiológico del bienestar. Terapia psicológica, 30, 77-84.

Verghese, J., LeValley, A., Derby, C., Kuslansky, G., Katz, M., Hall, C., y Lipton, R. B. (2006). Leisure Activities And The Risk of Amnestic Mild Cognitive Impairment In The Elderly. Neurology, 66, 821-827.

Wilson, R. S., Barnes, L. L., Aggarwal, N. T., Boyle, P. A., Hebert, L. E., de Leon, C. M., y Evans, D. A. (2010). Cognitive activity and the cognitive morbidity of Alzheimer disease. Neurology, 75, 990-996.

Wilson, R. S., Segawa, E., Boyle, P. A., Anagnos, S. E., Hizel, L. P., y Bennett, D. A. (2012). The natural history of cognitive decline in Alzheimer's disease. Psychology and aging, 27, 1008.

Wirdefeldt, K., Adami, H. O., Cole, P., Trichopoulos, D., y Mandel, J. (2011). Epidemiology and etiology of Parkinson's disease: a review of the evidence. European journal of epidemiology, 26, 1-58.

Zhang, X., Li, C., y Zhang, M. (1999). Psychosocial risk factors of Alzheimer's disease. Zhonghua yi xue za zhi, 79, 335-338. 
